\title{
BMJ Global Health Community health workers in Ghana: the need for greater policy attention
}

\author{
Leonard Baatiema, ${ }^{1,2}$ Anthony Mwinkaara Sumah, ${ }^{3}$ Prosper Naazumah Tang, ${ }^{3}$ \\ John Kuumuori Ganle ${ }^{4}$
}

To cite: Baatiema $L$,

Sumah AM, Tang PN, et al. Community health workers in Ghana: the need for greater policy attention. BMJ Global Health 2016;1:e000141. doi:10.1136/bmjgh-2016000141

Received 27 July 2016 Revised 31 October 2016 Accepted 3 November 2016

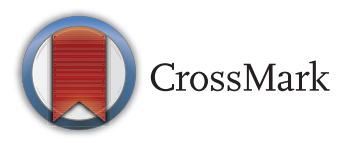

${ }^{1}$ Integrated Social

Development Centre, Tamale, Ghana

${ }^{2}$ Faculty of Health Sciences, School of Allied Health, Australian Catholic University, North Sydney, New South Wales, Australia

${ }^{3}$ Ghana Health Service, Upper West Regional Health Directorate, Wa, Ghana

${ }^{4}$ School of Public Health, University of Ghana, Accra, Ghana

\section{Correspondence to} Leonard Baatiema; baatiemaleonard@gmail.com

\section{ABSTRACT}

From the 1970 s to the 1990 s, the WHO, United Nations and other agencies mooted the idea of formally training and recognising community health workers (CHWs) to complement efforts to improve primary healthcare delivery in low and middle income countries. Recently, CHWs have been recognised as important players in the achievement of the healthrelated Millennium Development Goals (MDGs). Despite this recognition, little understanding exists in Ghana about the activities of CHWs: who they are; how they are recruited; what they do; level of health policy support; contribution to healthcare delivery and the challenges they face. Based on a rapid scoping review of the existing literature, and our experience working in Ghana, this paper reflects on the role of CHWs in healthcare delivery in Ghana. We argue that CHWs have played critical roles in improving health service delivery and outcomes, including guinea worm eradication, expanded immunisation coverage, maternal and child health, and HIV/AIDS treatment and management. However, these achievements notwithstanding, CHWs face challenges which prevent them from being optimally productive, including capacity problems, neglect by the healthcare system, high attrition rates and inadequate supervision. Policymakers in Ghana therefore need to give increased attention to CHWs, provide remuneration for their activities, create career opportunities and other means of motivations to boost their productivity and sustain gains associated with their activities.

\section{INTRODUCTION}

The concept and role of community health workers (CHWs) have enjoyed renewed interest in global health since the Alma-Ata Declaration in $1978 .^{1-6}$ CHWs have evolved over the past decades with its antecedence in the 'Feldshers' in 17th-century Russia, ${ }^{7}$ the barefoot doctors programme in China during the early $1920 \mathrm{~s},{ }^{8}$ and the WHO seminal work 'Health by the people' in $1975 .{ }^{9}$ Recent recognition that CHWs are important players in global efforts to achieve the health-related Millennium Development Goals (MDGs) highlights this attention. ${ }^{10}$ In 2013, the One Million CHWs Campaign

\section{Key questions}

What is already known about this topic?

- The contributions of community health workers (CHWs) to healthcare delivery have been recognised globally.

- There is a lack of global consensus on CHWs' scope of practice.

- There is inadequate health policy support for CHWs in low and middle income countries.

\section{What are the new findings?}

- This analysis presents systematic and comprehensive evidence of CHWs' contributions and health policy gaps in Ghana.

- It has contributed in clarifying the various classification of CHWs and their scope of practice.

- It has also highlighted evidence of inadequate health policy support for CHWs.

\section{Recommendations for policy}

- The formulation of a national policy on CHWs.

- The establishment of a professional and regulatory body to regulate the standards and practice of CHWs.

- The development of a long-term strategy that will see the gradual replacement or absorption of CHWs into the formal health service in Ghana.

project worth US $\$ 2.5$ billion was announced to boost the capacity of CHWs to deliver healthcare in sub-Saharan Africa. ${ }^{11}$ This current drive to recruit more CHWs is a recognition of their role in sustaining the MDG gains, as well as in ultimately achieving the more recent Sustainable Development Goals (SDGs).

A surge in the evidence of their contribution has recently been acknowledged in a Cochrane review on maternal and child health. ${ }^{12}$ Evidence in several low and middle income countries (LMICs) suggests that marked reductions in child and maternal mortality through health promotion interventions, immunisation campaigns and other community driven initiatives could be attributed in part to the role played by CHWs. ${ }^{11-15}$ 
With the current ageing population and wave of epidemiological transition globally (the impact of which is projected to be felt more by populations in LMICs), studies suggest that CHWs are rising to the task of ensuring that the impact is less felt by communities and health systems. ${ }^{16-18}$ The potential contributions of CHWs to tackling the current global health security threat posed by the Zika virus, yellow fever, Middle East Respiratory Syndrome (MERS) and influenza, especially in the area of surveillance, cannot be overemphasised.

Despite the general consensus about the importance of CHWs among the global health community, health policy interventions to recognise and support optimal delivery of healthcare by CHWs are lacking, especially in LMICs. In Ghana, although a number of studies and reports have highlighted CHWs' activities, ${ }^{16} 1920$ there is inadequate health policy support for them.

To the best of our knowledge, no studies currently exist in Ghana to have systematically profiled CHWs and their contribution to healthcare delivery. There is yet no consensus or comprehensive assessment of their roles, scope of activities and constraining factors to their productivity in Ghana. This analysis paper therefore addresses this knowledge and policy gap.

To analyse the activities of CHWs and the extent of health policy support in Ghana, we conducted a literature review of published and unpublished works, including reports on the activities of CHWs in Ghana (see box 1 for search strategy deployed to search and retrieve relevant literature for this study). This paper also incorporates insights from our experiences of working in Ghana as

\section{Box 1 Review approach}

A rapid literature review of both published and unpublished works was conducted in Academic Science Complete, CINAHL, Embase, Global Health Medline, Google Scholar, ISI Web of Knowledge, POPLINE PubMed, PsycINFO and Web of Science. No year limit was included in the search. We also undertook a review of reports on the activities of community health workers (CHWs) in Ghana. The search sought to retrieve relevant documents (programmatic and scholarly reports) on the activities of CHWs, especially in Ghana, using key words such as community health workers, CHW, village health worker, community health volunteer, lay health worker, traditional birth attendants, TBAs, Health Promoters, Ghana, remuneration, financing, health policy, impact, activities, training, practice and training. Relevant papers and information were retrieved and reference lists screened for more relevant studies. Studies which discussed the activities of CHWs with particular reference to Ghana were critically assessed for relevant information. In order to establish the activities of CHWs in the context of the globe, works related to CHWs in general were included based on relevance to the study aim. In addition, the scope of the literature included in this paper was confined to works which reported, described, analysed and synthesised the activities of CHW in the context of Ghana. Importantly, the paper incorporates insights on the activities of CHWs through anecdotes, authors' personal experiences and field reports. community health services researchers, affiliates of the Ghana Health Service and development workers who have contributed to the design and implementation of numerous community-driven health interventions in collaboration with CHWs.

\section{CHWs: evolution of the concept in Ghana}

\section{Categories of CHWs in Ghana}

Despite an international consensus about the importance of CHWs to healthcare delivery, a universal definition of CHWs remains evasive. Ambiguity further abounds in the mainstream literature on the characterisation of CHWs. ${ }^{21}$ However, during the recent United States Agency for International Development (USAID) CHW Evidence Summit, there was some consensus that a CHW is "A health worker who receives standardized training outside the formal nursing or medical curricula to deliver a range of basic health, promotional, educational, and mobilization services and has a defined role within the community system and larger health system". ${ }^{22}$ We find this definition to be insightful and thus will significantly support our analysis of CHWs in this paper.

In Ghana, two complementary types of CHWs are notable. The first category are an informal, poorly trained health service-supporting CHWs. In this category, CHWs are informally a part of the health system, and the health authorities or collaborating development partner (non-governmental organisation, NGO) takes responsibility for their recruitment, training, incentives and/or remuneration. The second strand of CHWs exists where CHWs are representatives of the community and act in the interest of the community. CHWs under this category have no or limited affiliation with the formal healthcare system, and mainly spearhead community-driven preventive health activities. Despite this distinction, we admit that many CHWs might be combining both roles in practice. From our experiences and in our view, a 'hybrid' form of CHWs (a mix of the two categories) can be found in Ghana.

Similar to the international trend, CHWs are diversely named in Ghana, including but not limited to names such as community health volunteers (CHVs), traditional birth attendants (TBAs), village health volunteers, community health champions, peer health educators, community child growth promoters, community nurses, community health committee members, community-based surveillance volunteers (CBSVs), community-based agents and lay health workers. ${ }^{16} 1920$ 23-26 ${ }^{20}$ These different characterisations generally reflect the diverse and ambiguous identities of CHWs in the international health literature and in health policy programmatic interventions. ${ }^{10} 2127-29$ For the purpose of clarity, we refer to CHWs in this paper as any of the above category of health workforce who, though not formal employees of the health system in Ghana, are either a health service-supporting CHW or community representatives, who have been identified, 
trained or untrained, and assigned roles in the community for which they receive no or some form of formal remuneration.

\section{Historical perspective of CHWs in Ghana}

Historically, CHWs such as TBAs have operated in most Ghanaian communities. ${ }^{30}$ However, attempts to formally organise and recognise them came after the Alma-Ata Declaration on Primary Health Care (PHC). Village health workers (VHWs) provided the first semblance of CHWs in Ghana. These were local community representatives whose actions and activities were mostly based on altruism. Although their roles such as community mobilisation, health awareness creation and immunisation campaigns were critical to the modest gains Ghana achieved from implementing the PHC strategy, their activities were short-lived such that by the 1980s, VHWs were no longer used. Lack of coordination and inadequate supervision of their activities were among the reasons why the VHWs programme was discontinued.

As a result, community health nurses (CHNs) were introduced to deliver healthcare in mostly deprived parts of Ghana. ${ }^{24}$ Unlike the VHWs, CHNs were recruited, provided professional training in nursing training colleges, deployed into mostly rural parts of Ghana and formally remunerated to provide healthcare. However, the relatively long period needed to train them and funding challenges limited adequate training and deployment of CHNs. Consequently, the activities of VHWs were reinvigorated in the early 1990s. Central to their re-emergence was the Navrongo Experiment, a pilot project which culminated in the current Community-based Health Planning and Services (CHPS) concept. ${ }^{24}{ }^{26}$ Since then, CHWs have continued to evolve both in name and role. Currently, CBSVs form the majority of CHWs in Ghana. However, there is still lack of clarity on their contribution, recruitment, scope of practice and remuneration.

\section{CHWs: contributions to healthcare in Ghana}

Evidence of the success of a plethora of community health interventions supported and delivered by CHWs exists in the international health policy and systems research literature. ${ }^{12} \quad 13 \quad 27 \quad 31$ Although CHWs are involved in many domains of healthcare delivery in Ghana, our review identified four important areas where CHWs' contributions have been immense. These four areas are examined in this paper.

\section{The Navrongo Experiment}

The Navrongo Experiment is one of the areas where CHWs' contributions to healthcare delivery have been acknowledged globally. The Navrongo Community Health and Family Planning Project, popularly referred to as the Navrongo Experiment, which culminated in the birth of the current CHPS programme in Ghana, sought to improve access to healthcare in deprived communities using mostly community-driven resources and structures. The intervention was conceptualised under the assumption that by recruiting, training and deploying community health officers to rural communities, inequities in access to healthcare services between urban and rural settings could be bridged. Under the Navrongo Experiment, CHWs played several critical roles, including outreach services, community mobilisation for health educational talks and referral to health facilities. ${ }^{32} 33$

The Navrongo Experiment resulted in increased access to healthcare services, immunisation coverage, reduced child and maternal mortality while improving the rural population's overall health. For example, within a period of 3 years of its implementation, the results pointed to a $50 \%$ reduction in childhood mortality rate and $15 \%$ reduction in fertility rate. ${ }^{24}$ Given this landmark success, results from this experimental intervention led to the conceptualisation and implementation of the CHPS programme as the country's main policy enactment aimed at improving access to health services in rural Ghana. Owing to its overall success, Ghana's CHPS programme has been acclaimed as among the most successful community-based health programmes globally. ${ }^{32-35}$

\section{Guinea worm eradication}

Without the diverse contributions of CBSVs, Ghana's current success in eradicating the guinea worm (Dracunculiasis) would have been unlikely. Ghana was ranked second globally in 2006 among the guinea worm endemic countries. ${ }^{36}$ Currently, however, Ghana is guinea worm free. Studies and reports from the Ghana Health Service and non-state actors (NGOs) have underscored the critical roles played by CHWs in eradicating the guinea worm in Ghana. ${ }^{38-41}$ Their roles included community mobilisation for awareness raising campaigns on the spread of the guinea worm, administration of palliative care, door-to-door distribution of drugs, referral to health centres for treatment, and distribution of water filters in communities. ${ }^{38} 394243$ Indeed, their role was so critical that both the National Guinea Worm Eradication Programme and some development partners (eg, Carter Center) have acknowledged it as being the most important factor in Ghana's guinea worm eradication campaign. ${ }^{44}$

\section{Promotion of maternal and child health}

Globally, CHWs have been acclaimed as having played a tremendous role in decreasing childhood illnesses and mortalities. ${ }^{13} 4546$ Notably, case management of childhood illness is one area where their contribution has been highly commended by the WHO and UNICEF. ${ }^{47} \mathrm{~A}$ study in Ghana reported that CHWs were instrumental in establishing community health post, making home visits to provide healthcare services such as administration of antibiotics, oral rehydration solution and zinc to treat childhood illnesses such as pneumonia, diarrhoea and malaria. ${ }^{48}$ Similar results have been reported by 
other studies globally. ${ }^{20} 23244950$ Again in Ghana, the role of TBAs has been reported to double the number of women referred to clinics and hospitals for potentially life-saving care and support. ${ }^{51}$ Indeed, in several communities in Bolgatanga, Kassena Nankana and Bawku West districts of Ghana, Oxfam has trained and evaluated the work of some 150 TBAs. ${ }^{30} 51$ The evidence from Oxfam's work has shown positive results. In each of the communities where Oxfam trained and worked with TBAs, the number of women being referred by TBAs to clinics and hospitals for potentially life-saving care doubled. ${ }^{51}$ Maternal mortality has similarly reduced by $7 \% .^{51}$

Further, between 2005 and 2007, one of the authors (JKG) was involved in a community-based pilot health project in 30 communities in Nadowli District, Upper West Region, Ghana. ${ }^{30}$ This project was implemented by World Vision Ghana in partnership with Ghana Health Services. The project trained CBSVs and TBAs to perform a number of tasks, including recording births and deaths, and reporting disease outbreaks. ${ }^{30}$ Several TBAs also got trained to detect danger signs during pregnancy and labour and to make quick referral of pregnant women to health facilities to receive skilled care. ${ }^{30}$ The TBAs were all provided basic consumables such as hand gloves, hand sanitisers, new packs of cutting blades and kerosene lanterns (to be used in the night when there is no electricity). The main aim of the project was to improve TBAs' skills and resource them adequately to conduct normal deliveries, particularly in hard-to-reach rural communities. In mid-2007, an initial evaluation was done. The evaluation results showed that antenatal care attendance in some communities had increased twofold (ie, $41 \%$ in 2005 to $85 \%$ in 2007). ${ }^{30}$ Qualitative interviews with women and TBAs suggested that many TBAs who received the training and essential consumables actively encouraged and referred pregnant women to healthcare facilities. ${ }^{30}$ Also, among women who delivered at home with TBAs, infections resulting from the use of bare hands and other unhygienic practices by TBAs (such as the same blade being used to sever the umbilical cords of two babies) during labour were reported to have reduced. The women and TBAs who participated in the interviews largely attributed the increase in the number of referrals to health facilities for skilled delivery and a reduction in infections during labour to the training TBAs received and the supply of hand gloves, hand sanitisers and new blades. ${ }^{30}$ In fact, these positive results from Ghana are supported by evidence from other low income contexts. ${ }^{52-56}$ Notwithstanding these evidences, TBAs' activities in maternal healthcare until now are still surrounded with controversies because the WHO official position only permits TBAs to make referrals and not conduct home deliveries. ${ }^{30}{ }^{51}$ However, based on this evidence from the Upper East Region and the fact that in Ghana only $55 \%$ of births are attended to by a skilled attendant (defined here as a doctor, nurse or midwife), and $30 \%$ by TBAs, ${ }^{30}$ we consider the
WHO position on TBAs as untenable in LMICs such as Ghana where the capacity to provide skilled and supervised delivery is limited.

\section{HIV/AIDS treatment and management}

In other parts of the world especially in sub-Saharan Africa, the role of CHWs has been important in the fight against the spread of HIV/AIDS. ${ }^{57-60}$ In Ghana, the literature suggests that CHWs were recognised as a conduit to providing voluntary counselling and testing services. A cross-sectional survey conducted in northern Ghana reported an overwhelming acceptance (98.7\%) of the use of lay health counsellors to provide community-based voluntary counselling and testing services. ${ }^{19}$ We noted, however, that only a few studies have covered the activities of CHWs in HIV/AIDS prevention and treatment in communities. Our experience in working at the community level in Ghana shows that CHWs are a key cadre of health staff working closely with CHOs and NGOs in raising HIV/AIDS awareness, mobilising communities against stigma and discrimination and providing a culturally acceptable or a community-competent context for people living with HIV/AIDS. Despite this, the District Health Information Management System (DHIMS), a comprehensive database which reports on all aspects of services delivered by the Ghana Health Service, does not report on CHWs' contribution to HIV/AIDS prevention and management. This suggests that even in contexts where their contribution is not in doubt, the healthcare system has not adequately documented or recognised their role.

\section{Lack of policy clarity on CHWs in Ghana}

\section{Recruitment}

Prior to the inception of the One Million CHW Campaign (to which we return later), there was no national framework to guide the recruitment of CHWs in Ghana. As a result, their recruitment was dependent on the community, the health programme, and the donor agency or development partner (NGO) involved. From the international literature ${ }^{48} 50$ and our experiences in Ghana as well, CHWs are generally recruited from their own communities based on their level of acceptability in their respective communities, previous involvement in community-driven initiatives, high sense of dedication to duty and literacy (education) level. Although these considerations are important, they are no doubt subjective and may affect the objective assessment and recruitment of CHWs. Our experience in some communities shows that the lack of clearly defined recruitment criteria and strategy has often generated tensions among community members. Indeed, some earlier studies have indicated how recruiting CHWs from local communities often served to generate tension and/or perpetuate gender inequalities. ${ }^{61}$

An important dimension regarding the recruitment of CHWs in Ghana is the active role of NGOs and other non-state actors. Many NGOs and other non-state actors 
who operate and/or implement parallel or vertical healthcare programmes in Ghana often recruit parallel CHWs. In most cases, this parallel recruitment is often without regard for existing structures. From our experience, the NGOs' style of engagement with CHWs can be problematic because recruiting CHWs without recourse to already existing structures can breed conflicts, duplication of efforts, lack of community participation and ownership of a particular health intervention and redundancies following the completion of the programmes of such organisations.

\section{Scope of practice}

Comparable to the global situation, the scope of practice for CHWs lacks clarity and remains undefined in Ghana. There has been a long-standing debate as to what exactly their roles should be and to what extent they are supposed to act in providing healthcare. ${ }^{21}{ }^{62}$ According to a report by UNICEF, in South Asia, one CHW can provide healthcare services to about 10 to 1000 households. ${ }^{63}$ Generally, CHWs tend to provide more preventive and promotive healthcare, rather than curative. ${ }^{11} 27$ In other settings, the roles of CHWs have been observed to vary from community mobilisation for immunisation campaigns, health talks, first aid, creating awareness on disease control and health promotion to activities such as registration of births and deaths. ${ }^{12} 212764$

In Ghana, owing to the acute shortage of health personnel, CHWs' scope of practice has broadened beyond prevention and health promotional activities to encompass some curative care such as treatment for malaria and diarrhoea. Generally, the scope of practice of CHWs in Ghana varies widely, including serving as aids to community health officers, home visits, disease surveillance, maintaining environmental sanitation, nutrition education, home management of minor ailments like uncomplicated malaria, social mobilisation, and providing a limited range of reproductive and child health services. ${ }^{38} 65$ While this varied and flexible scope of practice may be a unique strength, it is therefore to be inferred that the lack of clarity on the operational mandates of CHWs in Ghana has often undermined the effectiveness and efficiency of their roles in healthcare delivery at the community level. As observed by some researchers, a reasonable involvement of CHWs in limited activities has the tendency to enhance outcomes in community-based health interventions compared with an unlimited scope of practice. ${ }^{31}$

\section{Remuneration}

Remuneration of CHWs is a controversial subject that has eluded consensus among stakeholders. ${ }^{38} 66$ Internationally, the discourse is suggestive of the need to compensate their services in the form of a fee or in kind by the beneficiary communities. Some other nonmonetary incentives such as providing bicycles, certificates or free healthcare to CHWs are also common. ${ }^{316768}$ As a result, the WHO has underscored the need to pay CHWs reasonable wages in order to enhance their productivity, sustain community-driven interventions and reduce their attrition rates. ${ }^{69-71}$ The available literature has copiously cited the positive correlation between incentivising CHWs and lower rates of attrition. ${ }^{13} 215772$

In Ghana, CHWs are not remunerated by governments, ${ }^{48}$ and a spirit of volunteerism and altruism is rather emphasised. CHWs are required to draw satisfaction from community recognition, ability to gain skills and experience and the opportunity it presents to them to build social capital and access other job opportunities. This contradicts the WHO position and existing literature, which recommend the remuneration of CHWs. ${ }^{73}{ }^{74}$ For instance, in the work of Lehmann and Sanders, ${ }^{21}$ the non-payment of CHWs under the premise that they were volunteers and offered services based on altruistic motives failed to motivate the CHWs to support the sustainability of community-based health interventions. Our experience suggests that contrary to the government's view that CHWs activities should be inspired by altruism, CHWs usually have high expectations of rewards in the form of regular wages, stipends and some form of career opportunities to eventuate them into the health system.

\section{CHWs: challenges and recommendations in Ghana}

While CHWs are gaining increasing attention as important players in healthcare delivery in Ghana and elsewhere, they are beset with multiple challenges. CHWs face capacity problems as they receive little or no formal training. One author noted this as a fundamental concern among CHWs as most of the experience they require for their job are gathered on the job. ${ }^{4}$ Quite notably, the Ghana Health Service recently attempted to address this challenge by developing training guides for some categories of CHWs. ${ }^{65}$ However, from our experience, there has been limited uptake of these training guides. We have also observed the inadequate supply of basic equipment such as wellington booths, bicycles, hand gloves and the first aid kit that some CHWs use to facilitate their work.

Neglect or inadequate recognition of CHWs is another fundamental challenge. As noted earlier, the absence of a policy directive on CHWs lends credence to our position. The current policy position of the government, which does not remunerate CHWs for contributing to deliver community health service, only exacerbates the dwindling enthusiasm of CHWs and the time they commit to their duties. This, to a large extent, affects negatively their productivity and retention. Further, this has the potential to increase their attrition rates as has been reported in other settings to vary between $3.2 \%$ and $77 \% .{ }^{13} 5075$ Also, the attendant long-run effect of this situation is the high costs involved in selecting and training new CHWs as replacements.

The inadequate recognition of CHWs has further led to the lack of a framework to regulate their practices as 
evidenced in the varying roles CHWs assume as espoused in earlier sections of this paper. The situation creates an inherent ambiguity and varying expectations of their roles which affects the optimum engagement of CHWs. In Ghana, there are neither mechanisms nor a framework to regulate their practice or certify CHWs as having the requisite competencies to practice as in the case of other health professionals such as medical doctors, nurses or CHNs. To be able to attain standardisation and integration of the services of CHWs into the health system, a form of oversight is required. This may take the form of a regulatory or professional body which will provide some form of certification or licensing to duly recognise their competencies and standardise their practices.

Lack of effective supervision of CHWs is one of the notable problems CHWs face in Ghana. Our experiences in northern Ghana suggest that laxity in the supervision of CHWs is mainly due to the already overstretched nature of the health system, which is attributable in part to the human resources for health crises. Given that CHWs are now taking up more curative care activities in addition to the preventive-based care interventions, their activities should be supervised and coordinated closely to potentially optimise their productivity and improve health outcomes as evident in studies from other settings. ${ }^{64} 7677$

Also, a further step by the Ministry of Health $(\mathrm{MOH})$ to scale up the number of frontline staff to provide healthcare to the rural communities is the current partnership with the One Million CHW Campaign project to introduce a new cadre of CHWs in Ghana. This represents an important health policy direction in improving access and health outcomes of the rural populace in Ghana. The One Million CHW Campaign proposed to introduce a world-class cadre of CHWs to extend essential health services to household levels functioning as an integral component of the community health system. ${ }^{78}$ Conceptually and based on the literature on CHWs, this category of CHWs will be an informal extension of the formal healthcare system with explicitly defined roles, training and remuneration to augment the delivery of healthcare. Notably, this new cadre of CHWs differs substantially from the CHWs who are the subject of this paper except in their duties. While the policy issues of existing CHWs still remain to be addressed, the new programme only proposes to recognise the existing CHWs provided they will be useful in providing voluntary assistance to the formally remunerated new cadre of CHWs, and their continuous existence will be dependent on the particular health district. A justifiable policy question may therefore be why introduce a new cadre of CHWs to take up duties which were essentially carried out by a particular workforce but whose contribution has hitherto not been recognised and whose integration into the new programme has not been explicitly addressed. If such concerns are not addressed, there will most likely be challenges such as conflicting roles, tension between CHWs of the One Million CHW Campaign and existing
CHWs, lack of community participation and a threat to the sustainability of community-based health interventions.

The preceding analysis points to a general policy deficit regarding CHWs in Ghana, given the lack of a national framework to guide activities of CHWs such as recruitment, credentialling, scope of practice, remuneration, career development, performance management, supervisory mechanisms, integration into the formal health system, capacity development and logistical requirement and deployment. Despite the active involvement of CHWs in the health system, they are invisible nationally and subnationally in terms of policies, strategies and budgeting. ${ }^{78}$ We therefore recommend the following immediate and long-term measures to forestall anticipated and existing challenges faced by CHWs:

1. The establishment or enactment of a national policy on CHWs which should define and outline the relevant aspects of their recruitment, credentialling, scope of practice, remuneration, career development, performance management, supervisory mechanisms, integration into the formal health system, capacity development and logistical requirement and deployment.

2. The establishment of a professional and/or regulatory body that will exercise oversight over CHWs by defining, monitoring or enforcing the standards and practice of CHWs in Ghana including licensing or a form of certification.

3. The development of a long-term strategy that will see the gradual replacement or absorption of CHWs into the mainstream or formal health service in Ghana.

4. That the proposed One Million CHW Campaign project should incorporate an integration plan aimed at enlisting existing deserving CHWs who have a wealth of experience and skill to their credit and to further develop the capacities of those with inadequate skills to enable them to enlist and participate in the programme.

5. Finally, policy steps should be taken to critically review the WHO position on TBAs' roles in promoting maternal health. Given the shortage of skilled birth attendants (SBAs) in Ghana and across sub-Saharan Africa as indicated by the latest WHO report on the 'state of the world's midwifery', ${ }^{79}$ it is clear that the ideal of ensuring skilled attendance at all births is not feasible or achievable in the short term. Therefore, reasonably acceptable equity and efficiency arguments can be made for the building of working partnerships with and incorporation of TBAs into the maternal healthcare system in contexts such as Ghana where skilled maternal healthcare provision is acutely limited.$^{30}$ Indeed, widespread collaboration through policy support for the healthcare system to identify, train and enhance the skill sets of TBAs is recommended. Partnerships between TBAs and SBAs would also be critical for helping healthcare workers to learn from TBAs how best to address the cultural 
needs and concerns of childbearing women. ${ }^{30}$ Thus, even if the Ghanaian health system were to train and deploy sufficient numbers of SBAs to all parts of the country in the future, TBAs could still play important roles in helping healthcare workers to provide culturally competent care. ${ }^{30}$

\section{Conclusion}

This relatively modest contribution by CHWs to healthcare delivery in Ghana suggests the need for state and non-state actors alike to build better working partnerships with CHWs, provide financial remuneration, create career opportunities and other means of motivations to boost their productivity and sustain gains associated with their activities. In addition, there is the need for policy definition to harmonise the issues relating to CHWs in general. This is because CHWs play an indispensable role in delivering health to their communities and assume and actively play the role of health activists and advocates. The reported challenges faced by CHWs can be addressed through appropriate national policy articulation, and therefore development partners should work in partnership with the government of Ghana to put in place these policies.

\section{Handling editor Douglas Noble.}

Contributors LB and AMS conceived the study. LB, AMS, PNT and JKG searched the literature. LB drafted the manuscript. All authors contributed substantially to the review of the manuscript for critical and intellectual content. All authors have read and approved the final version of the manuscript for publication.

Competing interests None declared.

Provenance and peer review Not commissioned; externally peer reviewed.

Data sharing statement No additional data are available.

Open Access This is an Open Access article distributed in accordance with the Creative Commons Attribution Non Commercial (CC BY-NC 4.0) license, which permits others to distribute, remix, adapt, build upon this work noncommercially, and license their derivative works on different terms, provided the original work is properly cited and the use is non-commercial. See: http:// creativecommons.org/licenses/by-nc/4.0/

\section{REFERENCES}

1. Standing $\mathrm{H}$, Chowdhury AMR. Producing effective knowledge agents in a pluralistic environment: what future for community health workers? Soc Sci Med 2008;66:2096-107.

2. Christopher JB, Le May A, Lewin S, et al. Thirty years after Alma-Ata: a systematic review of the impact of community health workers delivering curative interventions against malaria, pneumonia and diarrhoea on child mortality and morbidity in sub-Saharan Africa Hum Resour Health 2011:9:27.

3. Werner D, Saunders D, Schweiger M. Questioning the solution: the politics of primary health care and child survival. BMJ 1997;315:555

4. Celletti F, Wright A, Palen J, et al. Can the deployment of community health workers for the delivery of HIV services represent an effective and sustainable response to health workforce shortages? Results of a multicountry study. AIDS 2010;24:S45-57.

5. Schneider $\mathrm{H}$, Hlophe $\mathrm{H}$, van Rensburg D. Community health workers and the response to HIV/AIDS in South Africa: tensions and prospects. Health Policy Plan 2008;23:179-87.

6. Tulenko K, Møgedal S, Afzal MM, et al. Community health workers for universal health-care coverage: from fragmentation to synergy. Bull World Health Organ 2013;91:847-52.
7. Kenyon VA. Feldshers and health promotion in the USSR. Physician Assist 1985:9:25-6.

8. Sidel VW. The barefoot doctors of the People's Republic of China. N Engl J Med 1972;286:1292-300.

9. Newell KW. Health by the people. Geneva: WHO, 1975

10. Bhutta ZA, Lassi ZS, Pariyo G, et al. Global experience of community health workers for delivery of health related Millennium Development Goals: a systematic review, country case studies, and recommendations for integration into national health systems. Global Health Workforce Alliance 2010;1:249-61.

11. Singh $P$, Sachs JD. 1 million community health workers in sub-Saharan Africa by 2015. Lancet 2013;382:363-5.

12. Lewin S, Munabi-Babigumira S, Glenton C, et al. Lay health workers in primary and community health care for maternal and child health and the management of infectious diseases. Cochrane Database Syst Rev 2010;(3):CD004015.

13. Haines A, Sanders D, Lehmann U, et al. Achieving child survival goals: potential contribution of community health workers. Lancet 2007;369:2121-31.

14. Lewin S, Babigumira S, Bosch-Capblanch X, et al. Lay health workers in primary and community health care: a systematic review of trials. Geneva: World Health Organization, 2006.

15. Jokhio $\mathrm{AH}$, Winter HR, Cheng KK. An intervention involving traditional birth attendants and perinatal and maternal mortality in Pakistan. N Engl J Med 2005;352:2091-9.

16. Abanilla PKA, Huang KY, Shinners D, et al. Cardiovascular disease prevention in Ghana: feasibility of a faith-based organizational approach. Bull World Health Organ 2011;89:648-56.

17. Norris SL, Chowdhury FM, Van Le K, et al. Effectiveness of community health workers in the care of persons with diabetes. Diabet Med 2006;23:544-56.

18. Brownstein JN, Chowdhury FM, Norris SL, et al. Effectiveness of community health workers in the care of people with hypertension. Am J Prev Med 2007;32:435-47.

19. Baiden F, Akanlu G, Hodgson A, et al. Using lay counsellors to promote community-based voluntary counselling and HIV testing in rural northern Ghana: a baseline survey on community acceptance and stigma. J Biosoc Sci 2007;39:721-33.

20. Agyei-Baffour P, Hansen KS, Browne EN, et al. The amount and value of work time of community medicine distributors in community case management of malaria among children under five years in the Ejisu-Juaben District of Ghana. Malar J 2012;11:277.

21. Lehmann U, Sanders D. Community health workers: what do we know about them? The state of the evidence on programmes, activities, costs and impact on health outcomes of using community health workers. World Health Organization, 2007;2:1-42.

22. Naimoli J, Frymus D, Quain E, et al. Community and formal health system support for enhanded community health worker performance. A US government evidence summit. Final report. 2012. https://www.usaid.gov/sites/default/files/documents/1864/ CHW-Evidence-Summit-Final-Report.pdf

23. Chinbuah MA, Abbey M, Kager PA, et al. Assessment of the adherence of community health workers to dosing and referral guidelines for the management of fever in children under 5 years: a study in Dangme West District, Ghana. Int Health 2013;5:148-56.

24. Phillips JF, Bawah AA, Binka FN. Accelerating reproductive and child health programme impact with community-based services: the Navrongo Experiment in Ghana. Bull World Health Organ 2006;84:949-55.

25. Acheampong T. Factors influencing sustainability of community-based health volunteers activities in the Kassena-Nankana East and West Districts of Northern Ghana University of Ghana, 2012.

26. Nyonator FK, Awoonor-Williams JK, Phillips JF, et al. The Ghana Community-based Health Planning and Services initiative for scaling up service delivery innovation. Health Policy Plan 2005;20:25-34.

27. Perry HB, Zulliger R, Rogers MM. Community health workers in low-, middle-, and high-income countries: an overview of their history, recent evolution, and current effectiveness. Annu Rev Public Health 2014;35:399-421.

28. Berman PA, Gwatkin DR, Burger SE. Community-based health workers: head start or false start towards health for all? Soc Sci Med 1987;25:443-59.

29. Prasad BM, Muraleedharan V. Community health workers: a review of concepts, practice and policy concerns. A review as part of ongoing research of International Consortium for Research on Equitable Health Systems (CREHS). 2007. http://www.hrhresourcecenter.org/hosted docs/CHW_Prasad_Muraleedharan.pdf

30. Ganle JK. Chasing out traditional birth attendants in Ghanaimplications for maternal and newborn health. J Global Health 2014;4:39-42. 
31. Strachan DL, Källander K, ten Asbroek AH, et al. Interventions to improve motivation and retention of community health workers delivering integrated community case management (iCCM): stakeholder perceptions and priorities. Am J Trop Med Hyg 2012;87 (Suppl 5):111-19.

32. Awoonor-Williams JK, Feinglass ES, Tobey R, et al. Bridging the gap between evidence-based innovation and national health-sector reform in Ghana. Stud Fam Plann 2004;35:161-77.

33. Phillips JF, Bawah AA, Binka FN. Accelerating reproductive and child health program development: the Navrongo initiative in Ghana. Population Council New York, 2005.

34. Baatiema L, Skovdal M, Rifkin S, et al. Assessing participation in a Community-based Health Planning and Services programme in Ghana. BMC Health Services Research 2013;13:233.

35. Awoonor-Williams JK, Vaughan-Smithb MN, Phillips JF. Scaling up health system innovations at the community level: a case-study of the Ghana experience. Soc Determinants Sex Reprod Health 2010;51:51-69.

36. Glenshaw MT, Roy S, Ruiz-Tiben E, et al. Guinea worm disease outcomes in Ghana: determinants of broken worms. Am J Trop Med Hyg 2009;81:305-12.

37. World Health Organization. Dracunculiasis eradication: ministerial meeting, Geneva, 16 May 2007. Wkly Epidemiol Rec 2007;82:236-7.

38. Dil Y, Strachan D, Cairncross S, et al. Motivations and challenges of community-based surveillance volunteers in the northern region of Ghana. J Community Health 2012;37:1192-8.

39. Solomon AW, Akudibillah J, Abugri P, et al. Pilot study of the use of community volunteers to distribute azithromycin for trachoma contro in Ghana. Bull World Health Organ 2001;79:8-14.

40. McDonald M, Hunter J, Diallo DD, et al. Innovative approaches to infectious disease prevention in women. Emerg Infect Dis 2004;10: e6.

41. Hochberg N, Ruiz-Tiben E, Downs $\mathrm{P}$, et al. The role of case containment centers in the eradication of dracunculiasis in Togo and Ghana. Am J Trop Med Hyg 2008;79:722-8.

42. Cairncross S, Braide EI, Bugri SZ. Community participation in the eradication of guinea worm disease. Acta Tropica 1996;61:121-36.

43. Tayeh A, Cairncross S, Maude GH. The impact of health education to promote cloth filters on dracunculiasis prevalence in the northern region, Ghana. Soc Sci Med 1996;43:1205-11.

44. Caldwell L. Ghana: guinea worm eradication program gets results in country Atlanta. USA: Carter Center, 2009. (cited 2015 13/09/2015) http://www.cartercenter.org/news/publications/health/guinea_worm publications/ghana-gets-results.html

45. Sirima SB, Konate A, Tiono AB, et al. Early treatment of childhood fevers with pre packaged antimalarial drugs in the home reduces severe malaria morbidity in Burkina Faso. Trop Med Int Health 2003;8:133-9.

46. Yeboah-Antwi K, Pilingana P, Macleod WB, et al. Community case management of fever due to malaria and pneumonia in children under five in Zambia: a cluster randomized controlled trial. PLOS Med 2010;7:e1000340.

47. Young M, Wolfheim C, Marsh DR, et al. World Health Organization/ United Nations Children's Fund joint statement on integrated community case management: an equity-focused strategy to improve access to essential treatment services for children. Am J Trop Med Hyg 2012;87(Suppl 5):6-10.

48. Daniels K, Sanders D, Daviaud E, et al. Valuing and sustaining (or not) the ability of volunteer community health workers to deliver integrated community case management in Northern Ghana: a qualitative study. PLOS ONE 2015;10:e0126322.

49. Ahorlu CK, Koram KA, Seake-Kwawu A, et al. Two-year evaluation of Intermittent Preventive Treatment for Children (IPTC) combined with timely home treatment for malaria control in Ghana. Malar $J$ 2011;10:127.

50. Abbey M, Bartholomew LK, Nonvignon J, et al. Factors related to retention of community health workers in a trial on community-based management of fever in children under 5 years in the Dangme West District of Ghana. Int Health 2014;6:99-105.

51. Oxfam. Improving Maternal Healthcare in Ghana and Beyond. Oxfam: Oxford, UK, 2012.

52. Sibley L, Sipe T, Koblinsky M. Does TBA training increase use of professional antenatal care services: a review of the evidence. J Midwifery Womens Health 2004;49:298-305.

53. Ofili A, Okojie O. Assessment of the role of traditional birth attendants in maternal health care in Oredo Local Government Area,
Edo State, Nigeria. J Community Med Prim Healthcare 2005;17:55-60.

54. Sibley LM, Sipe TA, Barry D. Traditional birth attendant training for improving health behaviours and pregnancy outcomes. Cochrane Database Syst Rev 2012;(8):CD005460.

55. Titaley CR, Hunter CL, Dibley MJ, et al. Why do some women still prefer traditional birth attendants and home delivery?: a qualitative study on delivery care services in West Java Province, Indonesia. BMC Pregnancy Childbirth 2010;10:43.

56. Ana J. Are traditional birth attendants good for improving maternal and perinatal health? Yes. BMJ 2011;342:d3310.

57. Hermann K, Van Damme W, Pariyo GW, et al. Community health workers for ART in sub-Saharan Africa: learning from experiencecapitalizing on new opportunities. Hum Resour Health 2009;7:31.

58. Kober K, Van Damme W. Scaling up access to antiretroviral treatment in southern Africa: who will do the job? Lancet 2004;364:103-7.

59. Callaghan M, Ford N, Schneider H. A systematic review of task-shifting for HIV treatment and care in Africa. Hum Resour Health 2010;8:8-16.

60. Mwai GW, Mburu G, Torpey K, et al. Role and outcomes of community health workers in HIV care in sub-Saharan Africa: a systematic review. J Int AIDS Soc 2013;16:18586.

61. Jobert B. Populism and health policy: the case of community health volunteers in India. Soc Sci Med 1985;20:1-25.

62. Gilroy K, Winch P. Management of sick children by community health workers. Intervention models and programme examples. Geneva, Switzerland: World Health Organization, Department of Child and Adolescent Health and Development. 2006:72.

63. UNICEF. What works for children in South Asia: community health workers. Kathmandu: UNICEF, 2004.

64. Sprague L. Community health workers: a front line for primary care? Issue Brief 2012;846:137-44

65. Population Council. Community Health Volunteers Training Manual. Accra. 2009

66. Kironde S, Bajunirwe F. Lay workers in directly observed treatment (DOT) programmes for tuberculosis in high burden settings: should they be paid? A review of behavioural perspectives. Afr Health Sci 2002;2:73-8.

67. Mathauer I, Imhoff I. Health worker motivation in Africa: the role of non-financial incentives and human resource management tools. Hum Resour Health 2006;4:24.

68. Glenton C, Scheel IB, Pradhan S, et al. The female community health volunteer programme in Nepal: decision makers' perceptions of volunteerism, payment and other incentives. Soc Sci Med 2010;70:1920-7.

69. Crisp N, Gawanas B, Sharp I. Training the health workforce: scaling up, saving lives. The Lancet 2008;371:689-91.

70. World Health Organization, PEPFAR and UNAIDS. Task shifting: rational redistribution of tasks among health workforce teams: global recommendations and guidelines. Geneva, Switzerland: World Health Organization, 2007.

71. Ofosu-Amaah V. National experience in the use of community health workers. WHO, 1983.

72. Chankova S, Muchiri S, Kombe G. Health workforce attrition in the public sector in Kenya: a look at the reasons. Hum Resour Health 2009;7:58

73. World Health Organization. Task Force for Scaling Up Education and Training for Health Workers. Global Health Workforce Alliance Scaling Up, Saving Lives Geneva. Switzerland: WHO Press, 2008.

74. Witter S, Kusi A, Aikins M. Working practices and incomes of health workers: evidence from an evaluation of a delivery fee exemption scheme in Ghana. Hum Resour Health 2007;5:2.

75. Walt G, Perera M, Heggenhougen K. Are large-scale volunteer community health worker programmes feasible? The case of Sri Lanka. Soc Sci Med 1989;29:599-608.

76. Ingram M, Schachter KA, Sabo SJ, et al. A community health worker intervention to address the social determinants of health through policy change. J Prim Prev 2014;35:119-23.

77. Brownstein JN, Hirsch GR, Rosenthal EL, et al. Community health workers "101" for primary care providers and other stakeholders in health care systems. J Ambul Care Manage 2011;34: 210-20.

78. Frontline Health Workers Coalition. A Commitment to Community Health Workers: Improving Data for Decision Making. USA, 2014

79. ten Hoope-Bender P, Campbell J, Fauveau V, et al. The state of the world's midwifery 2011: delivering health, saving lives. Internat $J$ Gynecol \& Obstet 2011;114:211-12 